\title{
Investigating mechanical damage mechanisms of tapestries displayed at different angles using 2D DIC
}

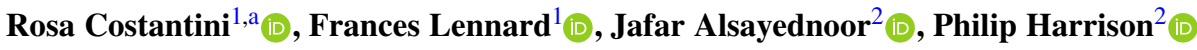 \\ ${ }^{1}$ School of Culture and Creative Arts, University of Glasgow, Glasgow G12 8QA, UK \\ ${ }^{2}$ School of Engineering, University of Glasgow, Glasgow G12 8QQ, UK
}

Received: 20 January 2020 / Accepted: 9 June 2020 / Published online: 22 June 2020

(C) The Author(s) 2020

\begin{abstract}
Tapestries represent a key but fragile component of many historic collections, as well as a complex challenge for textile conservators who aim to ensure their preservation. Indeed, the heterogeneity of materials, weaving features and conservation history make it difficult to predict the mechanical and physical behaviour of these objects, especially when hung for their display. The reported research postulates and investigates the role of different factors, namely relative humidity related strains and creep, in promoting the mechanical deterioration of tapestries. To track strain, the contactless full-field optical technique digital image correlation (DIC) was used. In particular, 2D DIC was successfully employed to monitor strains across large areas of a tapestry, demonstrating the occurrence of fatigue and creep damage mechanisms. While the former had the most influence on overall expansions and contractions, creep affected damaged areas. In addition, DIC was used for a preliminary evaluation of the efficacy of sloping boards, a non-traditional display method chosen by an increasing number of European museums in an attempt to reduce strain across tapestries. Both theoretical analysis and experimental work showed that, when minimal friction is present, small angles, like $5^{\circ}$ from the vertical, do not appear to promote noticeable reductions in strain.
\end{abstract}

\section{Introduction}

Historically, tapestries were the most widespread and explicit means to show off wealth and power by both religious and lay figures all across Europe [1]. Conservation is fundamental to preserve the cultural value of these works of art, which are known to be adversely affected by complex chemical and physical degradation processes [2]. However, there are no standard approaches among textile conservators [3]. Indeed, questionnaires conducted over the past three decades in both Europe and North America to investigate approaches in textile conservation show that, depending on the geographical area of the workshop and/or conservator's training, different methods are still used for treating tapestries [4-6].

\footnotetext{
a emails: r.costantini.1@ research.gla.ac.uk; costantini.rosa@gmail.com (corresponding author)
} 


\subsection{Damage mechanisms}

One of the main challenges in preserving tapestries is the mitigation of mechanically induced damage. Tapestries can be extremely large, with areal densities of around $1 \mathrm{kgm}^{-2}$ [7], or more. The self-weight of the tapestry generates significant loads along the weft threads, since the artworks are usually hung in this direction. This can be problematic as tapestries are weftfaced plain weaves, meaning that the weft threads create both the figurative design and the structural integrity of the object. Therefore, any mechanical damage in the weft direction can compromise the tapestry both structurally and artistically. Two plausible damage mechanisms are creep and fatigue.

Creep can be defined as a time-dependent deformation caused by an applied load [8]. To understand creep in tapestries, some explanation of their construction is required. A key feature is that weft yarns (largely made of wool and silk) are discontinuous as the threads change according to the colour pattern [9]. When the weft turns around the (usually wool) warp yarn, openings called slits are often formed (these were generally sewn together; however, the stitching could fail over time). Also because of this, the resulting weaving structure is highly heterogeneous. The ends of the open slits are prime locations for high stress intensity factors [10] and are likely sites for failure due to creep. In general, the actual threshold stress of the wool yarns depends on different factors, including the environmental conditions. When considering historic textiles, ageing and pre-treatments (e.g. dyeing methods) are also important variables [2].

A second potential damage mechanism is fatigue resulting from variations in humidity. Humidity may cause changes in both weight and swelling/contraction of the tapestry yarns [11-13]. Considering that wool and silk, the main components of tapestries, are hygroscopic materials, this is expected [14]. For instance, a recent article by Frame et al. demonstrated that tapestries temporarily expanded up to $6.5 \mathrm{~mm}$ as the relative humidity (RH) varied by around 30\% over 25 days [15]. Dynamically changing loads due to humidity-induced weight variations are one possible cause of fatigue, another is the internal stresses due to unequal swelling and contraction of the yarns. For example, if materials with different humidity related swelling/contraction responses are connected via stitching, this could create internal fatigue stresses, acting on the connective stitching. Since historic hangings may be displayed in uncontrolled environments, it is important to investigate the effects of fatigue alongside those of creep. A central objective in this investigation is to look for evidence for these two damage mechanisms.

\subsection{Damage prevention}

In an attempt to prevent damage, an increasing number of European museums and institutions employ sloping boards, also called slanted supports, for their display. One of the most renowned example is the Cluny Museum in Paris, where the famous The Lady and the Unicorn set is displayed on panels slanted at around $5^{\circ}$ from the vertical [16]. Use of slanted supports is a change in practice from the traditional approach, which usually consists of displaying tapestries vertically. This was historically achieved using rings, hooks and nails, though now less invasive hook-and-loop fasteners, such as Velcro ${ }^{\mathrm{TM}}$ tend to be employed [17].

The use of sloping boards was first promoted in the 1990s by André Brutillot, former private conservator and staff member of the Bayerisches National Museum in Munich (Germany). Brutillot suggested that this system enables better conservation since it can retard the elongation of weft threads by reducing self-weight loading. Aside from a couple of inter- 
national publications by Brutillot $[18,19]$, systematic studies on the actual efficacy of this display method have yet to be published. In a brief theoretical article, Barker considered the efficacy of slanted supports, calculating that the load experienced by fragile textiles could only be reduced by just $0.4 \%$ when using a support slanted at $5^{\circ}$ from the vertical, though crucially the effects of friction were omitted from this analysis [20]. On the other hand, the load could be more significantly eased by using greater slopes (e.g. $70^{\circ}$ from the vertical) [20, 21]. This, however, may pose curatorial and logistical issues.

It should be noted that slanted supports are often covered with textiles, such as cotton molton (domette) and polyester non-woven fabric, chosen in part to promote friction between the display board and tapestry [22]. Indeed, the effective coefficient of static friction between tapestries and these fabrics is reported to be very high $(\approx 1.5)$, when measured under an added normal load [23]. These first tests aiming to study friction between tapestries and the board covering fabrics can be found in Trosbach's dissertation (unpublished, in German) [23]. When displayed at an angle, this friction will inevitably decrease strain in the tapestry to some degree, where strain is the change in length per initial length [8]. However, it is important to distinguish the role of friction from that of the angle.

To start this investigation, we aimed to clarify the influence of both slope and friction on the self-weight loading of the tapestry and later measure its efficacy in reducing the two damage mechanisms proposed in Paragraph 1.1. To achieve these objectives, a combination of theoretical analysis and experimental measurements was employed.

\subsection{Digital image correlation for monitoring strain across tapestries}

DIC is a contactless optical technique that measures displacement and strain. This is done by taking successive images of the objects over time under various loading conditions and then analysing them through specific correlation-based algorithms. To allow correlation but also ensure accurate and precise data, the pattern on the specimens' surface requires certain qualities, such as: randomness, isotropy and high grayscale contrast. This is usually obtained by applying a high contrast random speckle pattern on the sample surface (e.g. black dots on a white background) [24]. Nonetheless, sometimes the natural texture of materials can be used in the DIC process with no need to apply a speckle pattern, as in the case of wood [25, 26].

A previous project carried out at the University of Southampton (2007-2010), the starting point of the current study, examined the viability of using 3D DIC in monitoring the strains occurring across tapestries [12]. In that study, the 3D DIC technique, which requires two cameras taking simultaneous pictures, was employed to monitor strains across a small area of actual tapestries (around $10 \mathrm{~cm}^{2}$ ) [11]. Initial results suggested that in some cases the intrinsic weaving pattern of tapestries can permit correlation. In addition, this previous research demonstrated that variations in strain were linked to changes in humidity levels [11, 12]. However, no in-depth studies were conducted to better distinguish the effects of different damage mechanisms, such as creep and fatigue, and therefore, further studies are needed.

This work investigates the feasibility of using DIC to monitor both local and global average strains occurring across large areas of historic tapestries. In doing so, the different mechanisms responsible for the potential mechanical degradation of tapestries are studied, namely: creep/uncrimping and fatigue. Uncrimping implies straightening out of initially nonlinear fibre paths under the action of gravitational loading and is difficult to differentiate from creep when measured using macroscale strain analysis. Strain monitoring begins after hanging the tapestries, and consequently, any preloading strain of the samples is omitted from the experimental measurements. Furthermore, DIC is employed to evaluate the efficacy 
of slanted supports as a display method, focusing on the role of inclination. In the present paper the 2D technique is used. 2D DIC requires only one camera; however, it cannot track out-of-plane displacements [24].

\subsection{Theoretical analysis of self-weight loading on sloping boards}

Prior to embarking on the experimental investigation, a theoretical analysis on the efficacy of sloping board for reducing strain across tapestry-like materials is presented. Due to the uncertainty in the thickness, $t$, of compressible fabrics, data is sometimes plotted as force per unit length, $N$, versus strain, rather than stress versus strain. The gradient of such experimental tensile test data is $E_{l}$, i.e. the sheet's tensile stiffness multiplied by its thickness, referred to here as the 'line stiffness' and can be a convenient property to use when characterising and modelling textiles $[27,28]$. Using a Coulomb friction model, the force per unit length acting along the top of the tapestry, $N(\mathrm{~N} / \mathrm{m})$, can be written,

$$
N=\rho g H(\cos \theta-\mu \sin \theta)
$$

where $\rho$ is the areal density of the fabric $\left(\mathrm{kg} \mathrm{m}^{-2}\right), g$ is the acceleration due to gravity $\left(\mathrm{ms}^{-2}\right)$, $H$ is the height of the tapestry $(\mathrm{m}), \mu$ is the coefficient of friction between the tapestry and the sloping board/covering material (-) and $\theta$ is the inclination of the display board from vertical $\left({ }^{\circ}\right)$.

Tests were carried out to study friction between tapestries (with and without linen support or cotton lining) and fabrics commonly employed by conservators for sloping boards (i.e. cotton molton/domette, polyester non-woven fabric). Measurements were conducted using a simple inclined plane method [29], both with and without a load (121 g) placed on the tapestry specimens. This method was preferred to other more elaborate techniques (e.g. [30]), since, especially without the extra weight, it better relates to actual conditions experienced by historic hangings displayed on a sloping board. When the weight was added, friction values were similar to those measured by Trosbach [23], i.e. around 1.5. However, results were found to be significantly higher without the load: tapestries showed no sign of slip even when raised to angles of $90^{\circ}$, meaning that, when interpreted using a simple Coulomb friction model, the coefficient is effectively infinite (or at least very high). This suggests that Coulomb friction is not actually representative of the slip behaviour of the textile, and so other forces are involved. For instance, it is thought that some kind of 'adhesion' may take place possibly due to interlocking of fibres or electrostatic interactions between the tapestry and the covering fabric [31]. More details on this series of tests can be found in Lennard et al. [32].

For the purpose of the simple mathematical analysis, this broad range of $\mu$ was considered in determining $N$ versus $\theta$, as shown in Fig. 1 (data normalised by the load at $0^{\circ}$ of inclination). Here the height of the tapestry is $2 \mathrm{~m}$ and the areal density is $1 \mathrm{~kg} \mathrm{~m}^{-2}$. As expected, Fig. 1 shows that the maximum line force experienced by the tapestry falls with both increasing inclination angle and with the raised effective friction coefficient. In the case of no friction, the load decreases are significant when sloping the display board at $45^{\circ}$, but very small for inclination angles of $5^{\circ}$ from the vertical or less. On the other hand, when examining a high coefficient of friction of 1.5 (as that calculated with extra weight in [23]), the reductions are already significant at angles of $5^{\circ}(\approx 15 \%)$ and fall to complete reduction of load at a display angle of about $34^{\circ}$. To reduce the load to 0 at smaller angles, such as $5^{\circ}$, the effective Coulomb friction coefficient would have to be close to 20 . However, as discussed before, this is not possible unless the presence of adhesive forces is postulated.

If the approximate material line stiffness of the tapestry is known, it is possible to estimate the strain profile from the bottom to the top of the tapestry (assuming for the sake of 


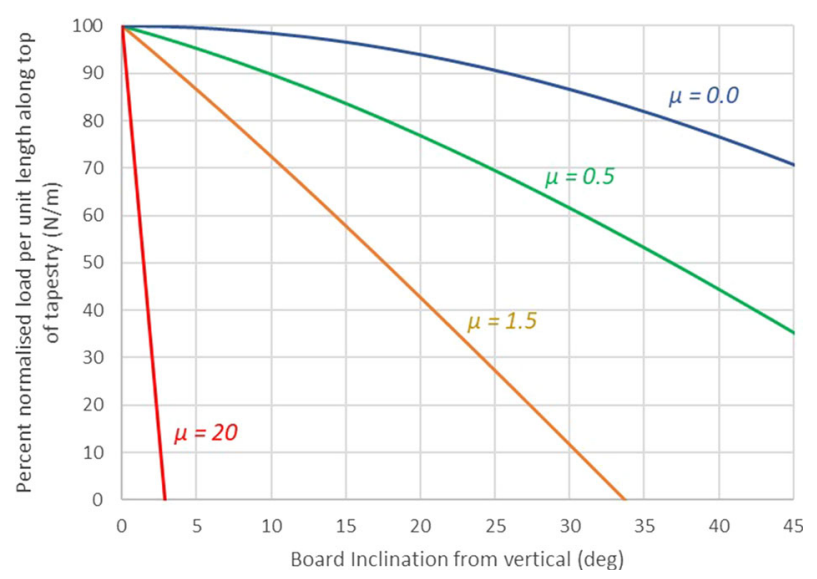

Fig. 1 Line force per unit length acting along the top of the tapestry versus inclination angle of the display board with different coefficients of friction, normalised by the load at $0^{\circ}$ of inclination

this calculation that the tapestry behaves as a homogeneous elastic sheet with no creep or viscoelastic behaviour). The strain can be plotted as a function of the height of the tapestry using Eq (2).

$$
\varepsilon=\int_{0}^{h} \frac{\rho g(\cos \theta-\mu \sin \theta)}{E_{l}} \cdot \mathrm{d} H
$$

where $h$ is any given height along the tapestry. Stress-strain data measured on actual tapestries can vary considerably in magnitude and usually shows strong nonlinearity at large strains, i.e. the line modulus changes with strain. As a first-order approximation the data can be fitted using an equation of the form

$$
E_{l}=a \varepsilon
$$

Substituting Eq (3) in Eq (4) find:

$$
\varepsilon=\sqrt{\frac{h \rho g(\cos \theta-\mu \sin \theta)}{a}}
$$

Using the data presented in Khennouf et al. [11], a 200,000 (N/m), this produces the strain versus height plots of Fig. 2, at different coefficients of friction but at a fixed inclination of $5^{\circ}$ from the vertical. Here $2 \mathrm{~m}$ corresponds to the top edge while $0 \mathrm{~m}$ is the bottom edge of the tapestry. Clearly, the greater the friction coefficient, the lower the strain experienced by the fabric; values of 20 or more lead to complete support ( 0 strain) in the tapestry.

While the theoretical results provide some enlightenment, this analysis does not consider the presence of heterogeneities (e.g. open slits) and only examines the effects of self-weight, omitting complexities introduced by more realistic material behaviour, like fatigue. Therefore, to have a better understanding of the mechanical responses in actual cases, real samples were tested as described in Sect. 2. 


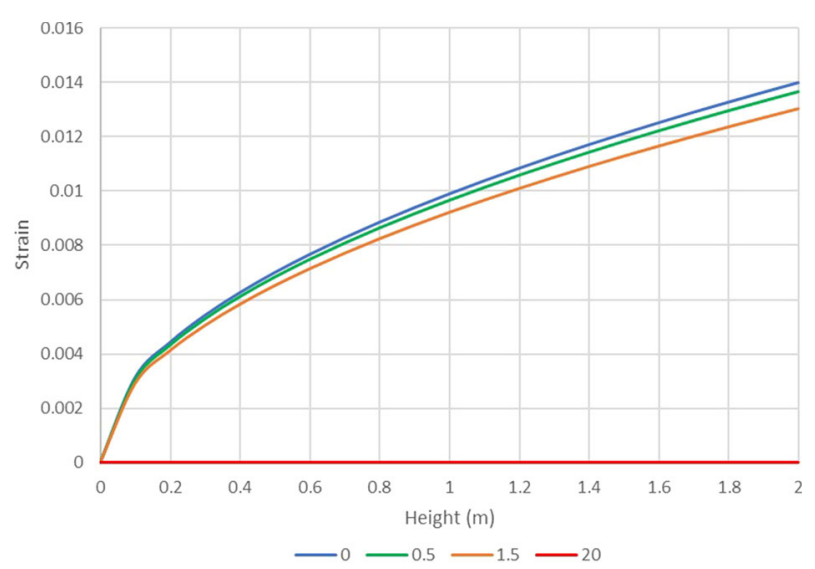

Fig. 2 Strain versus height of the tapestry when displayed $5^{\circ}$ from the vertical at different coefficient of friction: $0 ; 0.5 ; 1.5 ; 20$

\section{Materials and method}

\subsection{Historic fragment}

The strain across an historic fragment displayed vertically for $200 \mathrm{~h}$ was monitored by using 2 D DIC. The fragment $(\approx 160 \mathrm{~cm}$ high $\times 40 \mathrm{~cm}$ width) belongs to the Karen Finch Reference Collection at the Centre for Textile Conservation and Technical Art History. The object, made of wool in both warp and weft directions, was selected because of its weak condition, as evidenced by various open slits within its structure (Fig. 3). Because of this, the effects of creep and fatigue are expected to be significant and so more easily measured. The tapestry has a relatively low thread count of 4 warps $\times 14$ wefts per $10 \mathrm{~mm}$, that may also result in high strain when the fabric is subject to load (Fig. 4). The areal density of the tapestry is $1.14 \mathrm{kgm}^{-2}$.

\subsection{Fixed-load experiments with non-historic samples}

The evaluation of slanted supports was carried out using newly woven tapestry-like samples (Fig. 5). The aim was to mimic the mechanical behaviour of historic hangings, although simplifying it, as also done in other studies [11]. To accomplish that, a plain-weave, handwoven wool fabric was selected and used. The fabric, supplied by Context Weavers, has a thread count of 8 wefts $\times 23$ warps per cm (areal density $0.41 \mathrm{kgm}^{-2}$ ). The fabric was chosen because of its similar weave structure (shown in Fig. 6) and fibre composition to that of historic hangings. The wool rep is warp-faced, and not weft-faced like tapestries, but the warp threads of the plain-weave fabric show a comparable mechanical behaviour to that of weft threads of tapestries. The warp threads from the wool rep are tightly twisted, as are the weft ones of tapestries, while the plain-weave fabric weft threads are bulkier, like warps in historic hangings. Therefore, the wool rep specimens were hung in the warp direction. It should be underlined that a wool rep fabric was similarly employed by the research group at the University of Southampton, who also assessed the resemblance with tapestries in the tensile properties [11]. 
Fig. 3 Historic fragment, Karen Finch Reference Collection. Two open slits are highlighted in red
Fig. 4 Detail of the weave structure of historic fragment $(x$ $55)$. The vertical direction corresponds to the weft
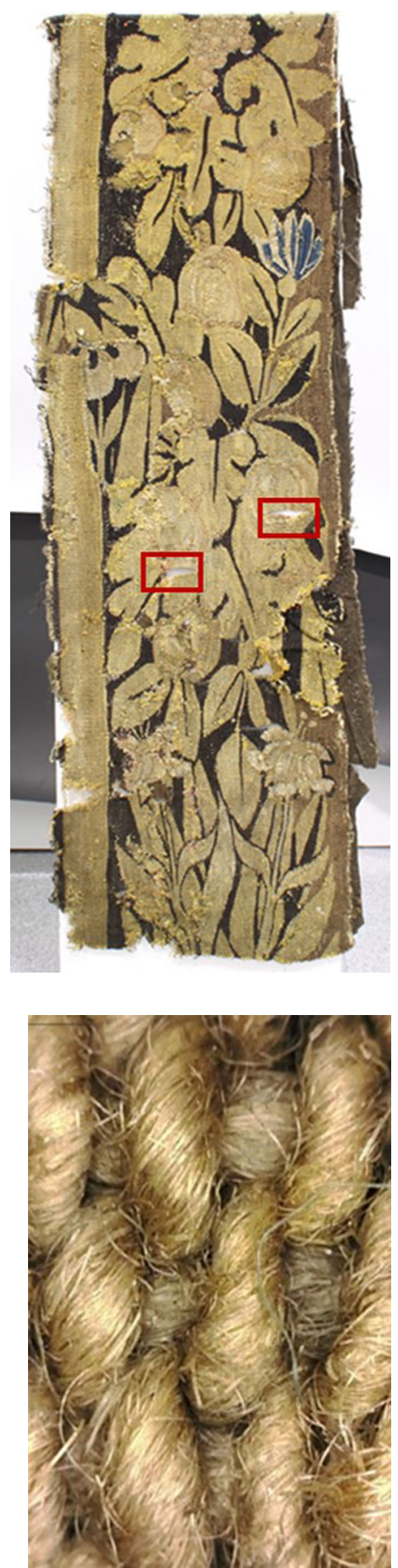




Fig. 5 Set-up of the wool rep samples on the board $\left(0^{\circ}\right.$ from the vertical)

Fig. 6 Detail of the weave structure of wool rep $(\times 55)$. The vertical direction corresponds to the warp

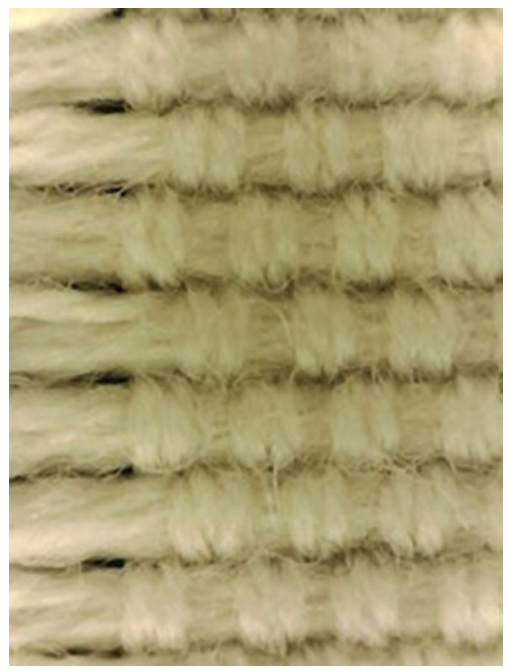

The samples were weighted to more closely match the loads expected near the top of a much larger and heavier tapestry. Each sample was loaded with $100 \mathrm{~g}$ of lead weights wrapped in a cotton strip stitched to the bottom edge of the sample, as similarly done in other studies testing conservation treatments [33-35]. For each test four samples were used, as illustrated in Fig. 5: three similarly damaged specimens and one undamaged specimen for reference. All the damaged samples had one central horizontal slit $50 \mathrm{~mm}$ long. The samples were $200 \mathrm{~mm}$ wide and $245 \mathrm{~mm}$ long (considering the area of interest of the DIC analysis). On the surface of the wool rep samples a speckle pattern with dots of around $1 \mathrm{~mm}$ in diameter was applied with markers to ensure the DIC correlation algorithm could track the strains.

\subsection{Digital image correlation analysis and monitoring set up}

Many factors can affect 2D DIC measurements, including experimental conditions (e.g. quality of the speckle pattern, image quality, out-of-plane displacements) and the settings used with the correlation algorithm [36]. To ensure good accuracy, the monitoring method 
was designed as follows. The strain monitoring of both the historic and model specimens was carried out by taking one picture per hour (automatically, using an auto shutter, to avoid any movement of the camera). The first picture was taken within around the first $10 \mathrm{~s}$ of the textile object being hung, in order to track displacement post self-weight loading. A Canon EOS $1000 \mathrm{D}^{\mathrm{TM}}$ (manual mode, no flash, $100 \mathrm{ISO}, \mathrm{f} / 8$ aperture, exposure time $1 / 4 \mathrm{~s}$ ) was employed. 2D DIC analysis was performed on the acquired images using Vic-2D 2009 by Correlated Solutions. A subset size of 61 and a step size of 5 were selected together with a non-incremental analysis. The Hencky strain was calculated using the DIC software and the data were then transferred to MATLAB to allow further analysis.

For the tests on the efficacy of slanted supports, a bespoke wooden (MDF) board adjustable to provide different display angles was designed and employed. Experiments with the mockups were repeated twice at: $0^{\circ}, 5^{\circ}$ and $45^{\circ}$ from the vertical. Monitoring at each inclination lasted $168 \mathrm{~h}$ ( 1 week). Whenever the angle of the board was changed, the camera was inclined accordingly, using a tripod, so that the sensor was always parallel to the monitored object. The textiles were attached to the board using a wooden batten (covered with Velcro ${ }^{\mathrm{TM}}$, soft side) which in turn was fixed at the top edge of the display board via four bar clamps. The board was left uncovered, minimising friction, and allowing a focus on the effects of inclination in reducing the strain. Still, the friction between the board and the wool rep is not insignificant: the coefficient of static friction is 0.6 (calculated according to testing method used in other studies [23]). However, considering the experimental conditions, the extra weight added to the specimens may have diminished the effects of friction. Besides enabling measurements at different angles, the board prevents out-of-plane displacements of the tapestry. During the experiments, a Hanwell Pro ML4000 RHT data logger was placed next to the monitored objects. The data logger was set up to record $\mathrm{RH}$ and temperature value every $15 \mathrm{~min}$. It was not possible to maintain constant temperature and humidity through the duration of the tests. However, while humidity fluctuated considerably, temperature remained stable $\left(20 \pm 2{ }^{\circ} \mathrm{C}\right)$.

\section{Results and discussion}

\subsection{D DIC analysis on a historic tapestry: what causes strain}

To better understand the different causes of strain, the tapestry fragment (see Sect. 2.1) was monitored for $200 \mathrm{~h}$ while hanging from a vertical board. The strain data were used to calculate both the average global strain across the entire specimen and the local strains occurring across and around damaged areas (slits) within the sample. It is important to emphasise that, since each slit gradually opened during the experiment, the high strain registered across the slit is not actual strain, rather it is an interpolated displacement across an expanding hole. The DIC algorithm effectively misinterprets the opening of the slits as a large positive strain. However, as long as the user is aware of this issue, the measurement can prove useful in monitoring the gradual opening of the slits. For simplicity, these misinterpreted local (hole) strains will be referred to henceforth as 'pseudo-strain'.

Figure 7 depicts both the globally averaged strain data in the vertical direction and RH data versus time. The graph shows that there is no direct proportionality between time and overall longitudinal strain, also indicated as $\varepsilon_{\mathrm{yy}}$. Instead, $\varepsilon_{\mathrm{yy}}$ correlates closely with changes in the RH levels, which varied between a minimum of $38.9 \%$ and a maximum of $59.7 \%$. Figure 8 demonstrates the good correlation between strain and $\mathrm{RH}$, as the coefficient of determination, $R^{2}$ (a numerical statistical measure to define the linear relationship between two variables), is close to $1(\approx 0.92)$. These reversible deformations in the vertical direction (both positive 


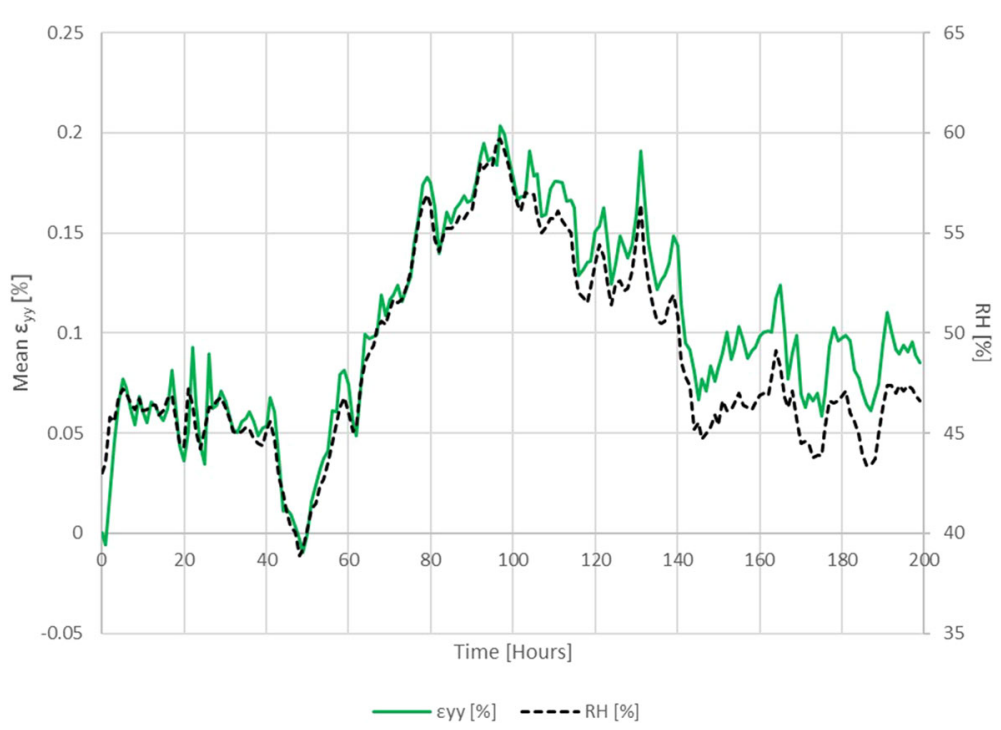

Fig. 7 Variations in the average longitudinal strain (green line) and RH (dotted line), during the 200-h monitoring

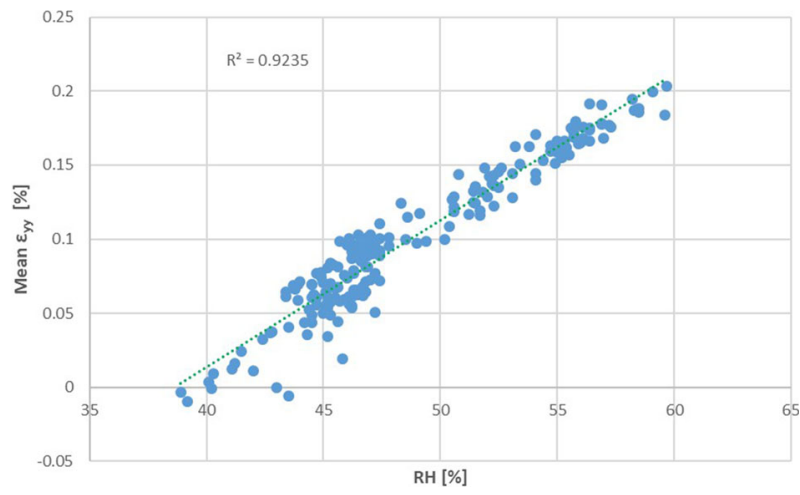

Fig. 8 Average longitudinal strain during the 200-h monitoring plotted against the changes in RH

and negative, indicating extension and contraction) can be partly due to changes in weight of the woollen fragment because of absorption/desorption of water from the environment and partly due to fibre swelling. It is important to note that the linear relationship between strain and relative humidity can only be observed within specific humidity intervals and under certain conditions. Indeed, the response of moisture content within natural fibres such as wool, to changing $\mathrm{RH}$, across the entire range, $0-100 \%$, is sigmoidal. In addition, the complex relationship between moisture uptake and humidity conditions is also linked to: (i) the difference in the rate of absorption and desorption; (ii) the hysteresis phenomenon; (iii) the time needed for the water content to reach equilibrium $[8,13]$.

From a quantitative point of view, the maximum longitudinal strain reached was around $0.20 \%$, which, considering the $1.3 \mathrm{~m}$ total length of the monitored area, indicates a longitudinal extension of $2.6 \mathrm{~mm}$. 
Fig. 9 Strain map of the historic fragment at the end of the $200-\mathrm{h}$ monitoring

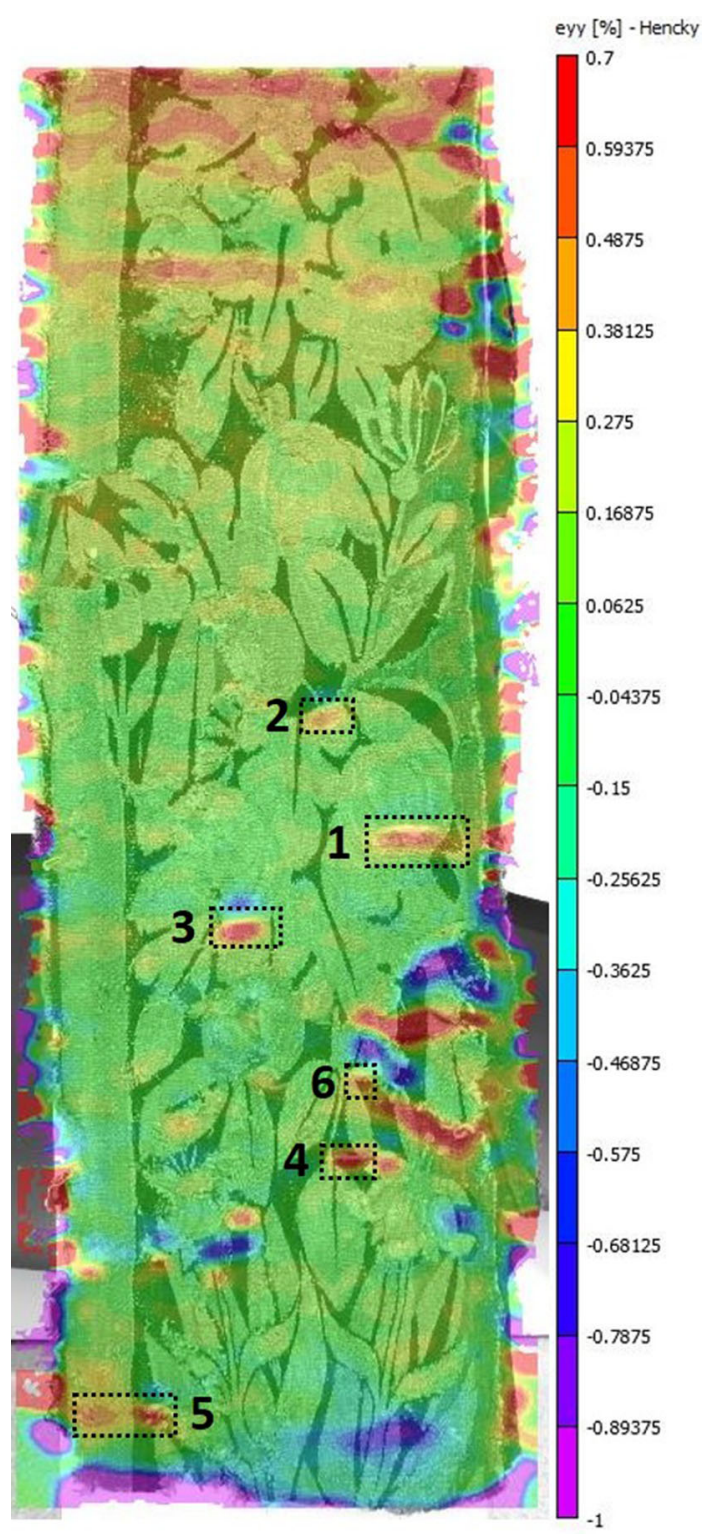

The data gathered showed that the tapestry also expanded and contracted in the horizontal direction. Like the longitudinal strain, mean $\varepsilon_{\mathrm{xx}}$ (horizontal strain) proved to be strongly correlated to changes in the RH level $\left(R^{2} 0.96\right)$, though here $\varepsilon_{\mathrm{xx}}$ is about half as large as $\varepsilon_{\mathrm{yy}}$. Because of this, it seems probable that dimensional changes caused by the varying RH levels can be linked to both fibre swelling effects and weight variations (though the latter only acts in the vertical, $y$, direction).

Strain maps, such as Fig. 9, reveal several areas of pseudo-strain at the location of the numerous slits present in the test piece, labelled 1-6 in the figure. This is a useful observation 


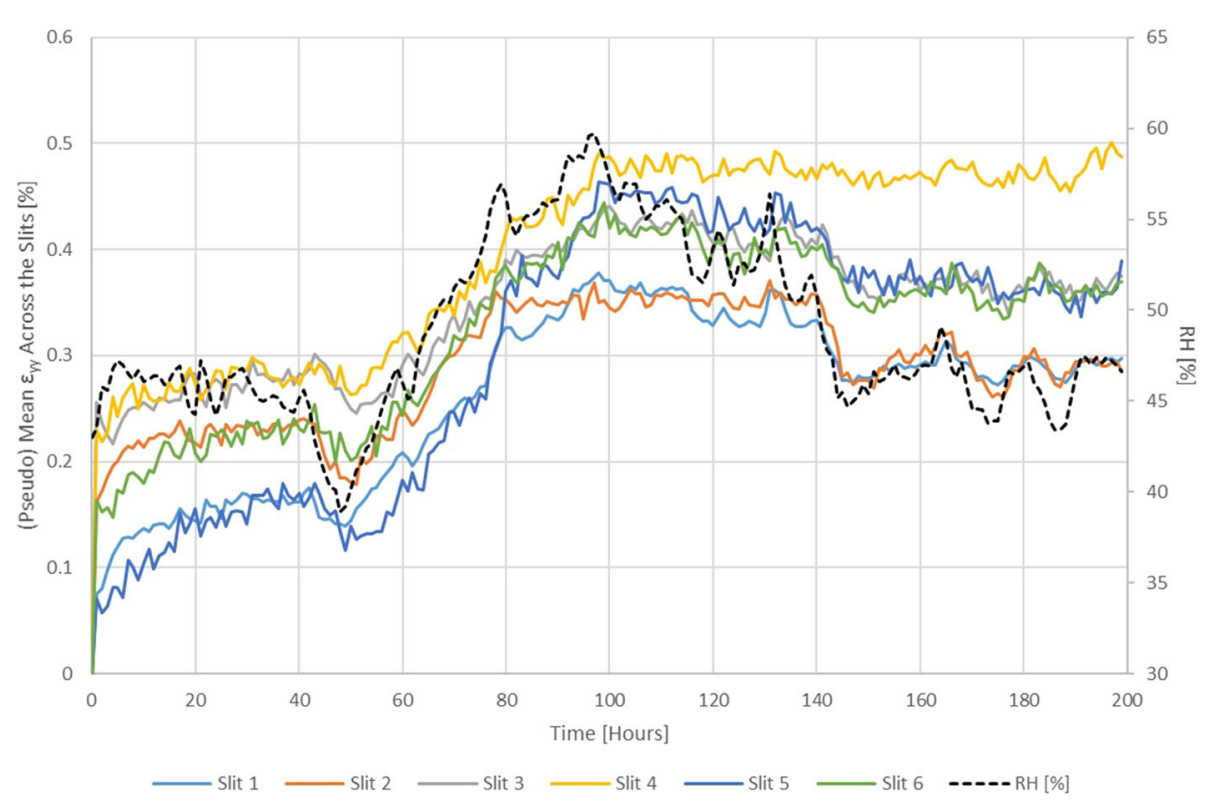

Fig. 10 The percentage pseudo-strain $\left(\varepsilon_{\mathrm{yy}}\right)$ acting across the slits versus time. RH values are also illustrated (dotted line)

since this research aims to monitor defects and study how conservation methods can be employed to prevent further degradation.

Besides the high pseudo-strain registered across the slit areas, it is apparent that the upper region of the fragment also demonstrates greater $\varepsilon_{y y}$ strains; this occurred during the entire length of the test. This type of strain profile is predicted in Fig. 2 for an elastic sheet initially unloaded, then subject to self-weight loading. However, given that the strain shown in Fig. 9 is measured post self-loading, another explanation is required. The cause behind this could be related to slight time-dependent uncrimping of the weft yarns. Besides, it should also be considered that the (local) slight non-planarity of the object may have also led to some errors. This can also explain the negative strain registered in a few specific points of the textile object, perhaps due to the local out-of-plane displacements of these extremely damaged (detached) areas.

It is interesting to note that the lower region of the tapestry showed more damages (open slits) than the upper one. Although it may be expected that damage would be more likely to occur higher up the tapestry because of the self-weight loading, other factors, characteristic of each historic hanging, also play a role, e.g. weaving mesostructure. In the case under investigation, the pre-existing conditions, and the visibly troubled conservation history, probably influenced the location of damages. In addition, the fragment presents a small textile patch on the top back that might have given some support to the upper region. Since the object is a fragment, it is underlined that the top does not correspond to the actual top of the whole tapestry and the distance between the upper and the lower part is relatively small.

Figure 10 presents graphs of the effective \% strain across the open slits plotted against time. By comparing the pseudo-strain data to the overall strain, it is observed that, as expected, the slits experience considerably greater pseudo-strain as they widen over time. The local pseudo-strain across the slits is found to depend less on the $\mathrm{RH}$ and more on time, in contrast 


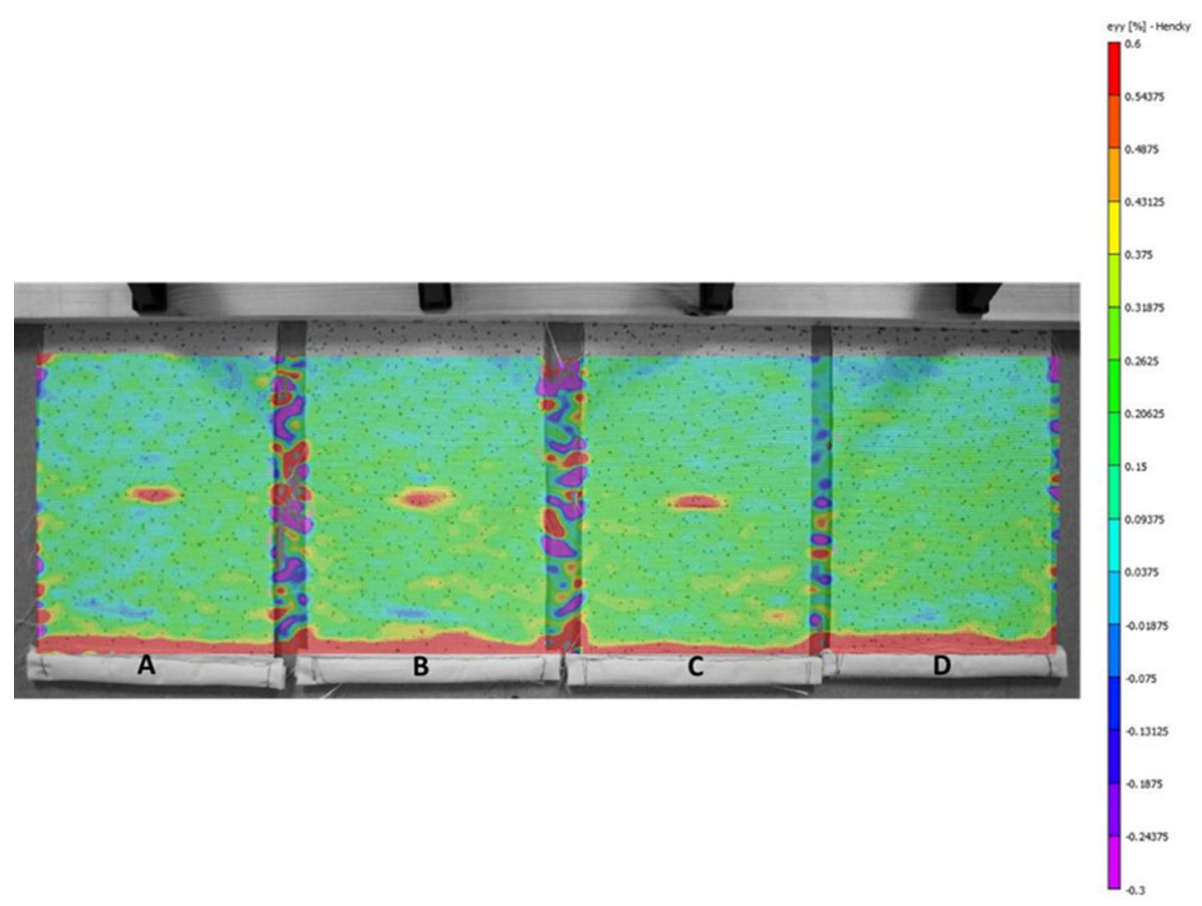

Fig. 11 Strain map of samples displayed at $0^{\circ}$ from the vertical after 1 week. Samples A, B, C have one central slit $5 \mathrm{~cm}$ wide, while sample D is undamaged

to the overall averaged strain data. This is not surprising as the effects of creep (or uncrimping) are expected to be greater in damaged areas due to stress concentrations occurring at the end of the slits. However, humidity still seems to play a role: between 50 and $100 \mathrm{~h}$ the humidity increased dramatically from $39 \%$ to $60 \%$, resulting in a steep rise in the pseudo-strain over the same period. In contrast with the overall strains, the slit widening is largely irreversible.

The 2D DIC strain monitoring of the historic tapestry fragment was successful in finding evidence for the mechanical damage mechanisms postulated in the introductory section: fatigue and creep. Nevertheless, the results demonstrate how difficult it can be to predict in detail where and how these mechanisms will develop. Factors such as the pre-existing condition of the textile object, but also uncertainties in capturing strain data due to the limits of the optical technique, make these outcomes only a first step towards a more comprehensive understanding of damage mechanisms affecting tapestries.

\subsection{Sloping boards: the role of inclination}

2D DIC was employed to monitor strain across wool rep samples displayed on an uncovered board slanted at $0^{\circ}, 5^{\circ}, 45^{\circ}$ from the vertical. This allows the further study, in actual cases, of the causes of strain in tapestry-like materials and the efficacy of (uncovered) slanted supports in mitigating them. Comparing the theoretical analysis of Sect. 1.4 to the experimental data presented here is also useful to better understand the validity of DIC for testing the effectiveness of conservation treatments.

Figure 11 shows strain maps across the model samples after being attached to the board $\left(0^{\circ}\right.$ inclination $)$ for 1 week, with a fixed load of $100 \mathrm{~g}$. The strain map reveals an area of 


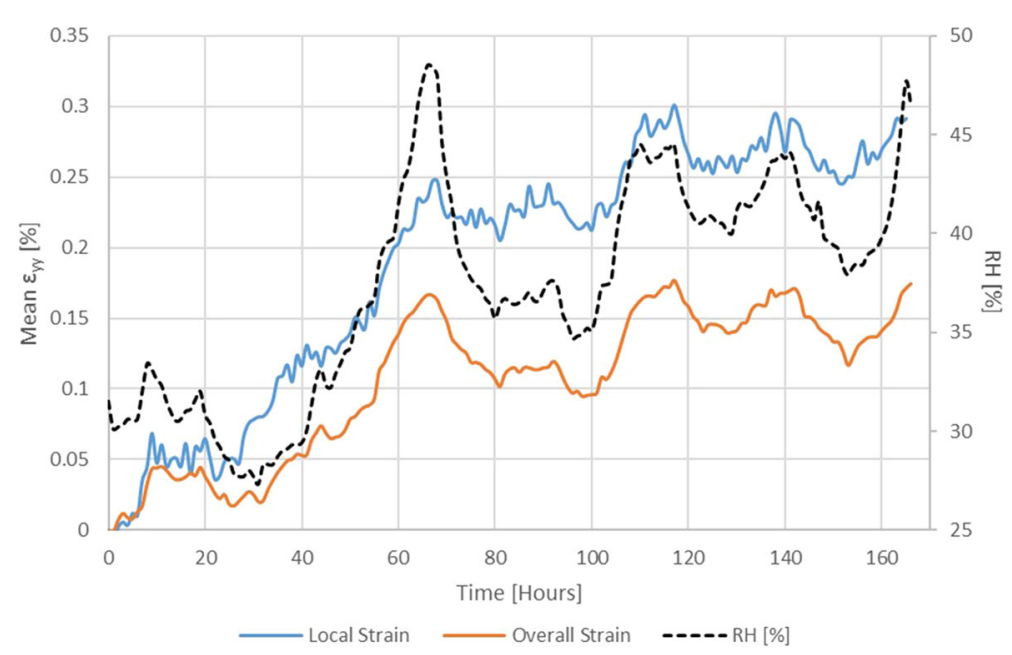

Fig. 12 Variations in the average longitudinal strain across the whole surface of damaged sample A (red line), and across the slit area (blue line) during the 1 -week monitoring at $0^{\circ}$ from the vertical. $\mathrm{RH}$ fluctuations are represented by the dotted line

pseudo-strain at the location of the central slit in each of the three damaged samples (A, B, C). Across undamaged areas, including the whole of sample $\mathrm{D}$, the strain is more homogeneous. In general, the data gathered from the analysis of the three damaged specimens is comparable. Note that the upper part of the samples was not analysed due to a shadow cast by the clamping batten, leading to inaccurate data. At the same time, data from the bottom areas were excluded from mean strain calculations since they showed unreliable values. This can be due to the non-planarity of the samples in the lower section, caused by the added loads.

Again, the average strain data from the overall area of the samples demonstrated close correlation with RH, as shown in Fig. 12. Therefore, fluctuations in RH (which in this test varied from a minimum of $27.3 \%$ to a maximum of $48.5 \%$ ) appear to be responsible for the majority of the average strain, either due to changes in weight or fibre swelling (for both $\varepsilon_{y y}$ and $\varepsilon_{\mathrm{xx}}$ strain).

Furthermore, Fig. 12 shows the pseudo- strain measured across the slit of sample A: here the mechanical deformation is dependent on both $\mathrm{RH}$ variations and time, suggesting that both weight change/swelling and creep (or uncrimping) play a significant role. Similar behaviour is reported in Sect. 3.1 on the historic fragment.

The influence of the environmental conditions on the averaged local strain data from samples displayed at different inclinations made it difficult to differentiate the role of display slope in mitigating creep and/or uncrimping. However, by comparing tests at $0^{\circ}$ and $5^{\circ}$ from the vertical, with similar humidity fluctuations, no obvious reduction in strain could be observed. Indeed, as shown in Fig. 13, the difference falls within the error in the measured data (averaged data from three damaged replicas of each experiment). In contrast, creep (or uncrimping) is reduced by about $40 \%$ for specimens displayed at $45^{\circ}$ regardless of the dramatic increases in humidity observed during the monitoring period. Given that Fig. 1 suggests a reduction in load of about $50 \%$ when the specimen is inclined at $45^{\circ}$, the outcome is reasonable.

Figure 14 illustrates pseudo-strain versus time of specimens monitored at $5^{\circ}$ and $45^{\circ}$, as well as the RH during the monitoring. The impact of humidity fluctuations is subtle but 


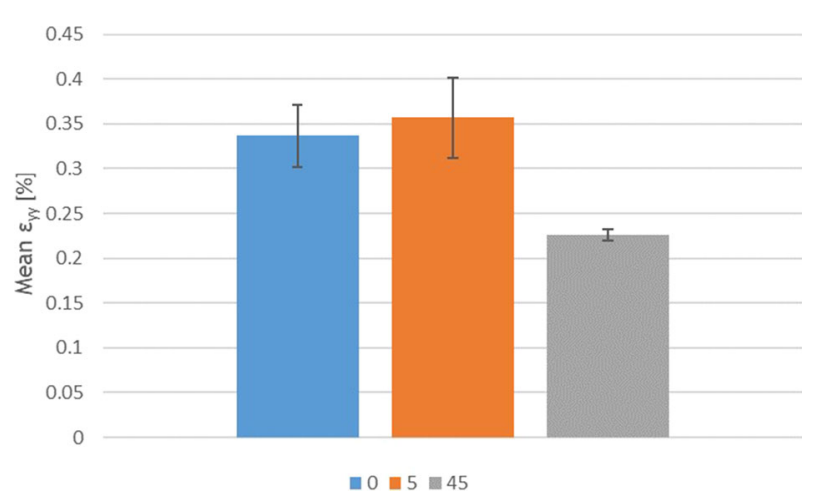

Fig. 13 Maximum values of mean pseudo-strain [\%] registered across the slits of the samples displayed at $0^{\circ}$, $5^{\circ}, 45^{\circ}$ from the vertical for 1 week

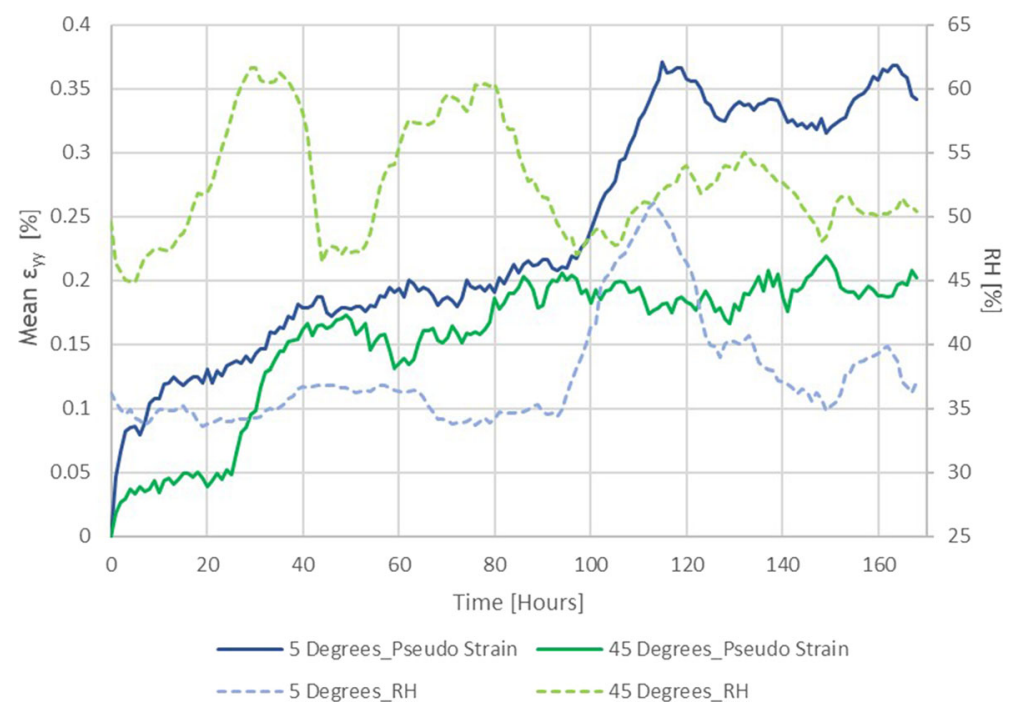

Fig. 14 Pseudo-strain across the slit area of samples displayed at $5^{\circ}$ and $45^{\circ}$ from the vertical (solid blue and green line) during the 1-week monitoring tests. $\mathrm{RH}$ variations during the experiments are represented by the dotted lines

evident: during the $5^{\circ}$ test $(86-113 \mathrm{~h}) \mathrm{RH}$ rose from 34.7 to $51.1 \%$, causing a steep increase in strain; during the $45^{\circ}$ test $(5-35 \mathrm{~h}) \mathrm{RH}$ raised from $44.8 \%$ up to $61.3 \%$, also resulting in an increase in strain. Still, at the end of 1 week of monitoring, the pseudo-strain was significantly greater for the samples displayed at $5^{\circ}$. The efficacy of the $45^{\circ}$ slope in easing the strain can be observed during the first $80 \mathrm{~h}$ of monitoring: while the local strain from the $5^{\circ}$ specimens reached a value of around $0.2 \%$ over a period with stable $\mathrm{RH}$, the $45^{\circ}$ sample reached a similar value only during an intense initial rise in $\mathrm{RH}$.

Overall, from a qualitative perspective, these results agree with the theoretical model, i.e. the beneficial effects of using uncovered display boards inclined by only few degrees (e.g. $5^{\circ}$ ) from the vertical is negligible, especially when the friction between the object and the board is low. Much greater angles, such as $45^{\circ}$, can promote strain reduction. While this 
is straightforward mechanical theory, it has not previously been explored in relation to the display of tapestries.

\section{Conclusions}

The current work postulated and investigated potential mechanical loading mechanisms experienced by tapestries when on display. The study examined the role of sloping boards in reducing the load on the tapestry using both theoretical and experimental approaches. The latter used 2D DIC to monitor strain, across both an historic fragment and wool mock-ups.

In the first part of the experimental study, post self-weight loading strains were measured across an historic fragment when displayed vertically for $200 \mathrm{~h}$. The 2D DIC strain maps successfully located weak areas (slits) via pseudo-strains. The latter were used to monitor slit widening over time. Averaged strain data (both $\varepsilon_{\mathrm{yy}}$ and $\varepsilon_{\mathrm{xx}}$ ) from the entire region of interest were shown to closely track $\mathrm{RH}$ variations: the random and high-frequency nature of these changing strains suggest a plausible mechanism for fatigue damage. Pseudo-strain data calculated across open slits increased during time, indicating a time-dependent creep-like behaviour. This could be due to gradual uncrimping of the highly crimped weft yarns or may possibly be revealing actual thread creep. Pseudo-strain was also partially influenced by RH fluctuations. To sum up, while fatigue has the most influence on a tapestry overall, damaged areas are more affected by (irreversible) creep. Similar mechanical behaviours were observed on wool rep mock-ups displayed on an uncovered board slanted at different inclinations (with relatively low friction). The data gathered also showed the efficacy of a $45^{\circ}$ slope, in contrast to a $5^{\circ}$ slope, in decreasing the strain, especially around weak areas (slits). The good correspondence of the DIC results with the theoretical model is important to assess the reliability of the technique to further test conservation treatments for textiles. Overall, the data from both the theoretical and experimental analysis have implications for tapestry display practice.

Importantly, 2D DIC was successfully used to measure strain across the overall surface of the historic fragment $(130 \times 40 \mathrm{~cm})$, demonstrating how the technique can be employed to study larger areas of historic hangings than those previously researched with the same method [11].

Acknowledgements This work was funded by the Leverhulme Trust (Ref: RPG-2015-179). The authors are very grateful to the textile conservators at Glasgow Museums, Maggie Dobbie, Helen Hughes, Harriet Woolmore, for their continuous support and guidance. In addition, the authors wish to thank Stephen McCann and Sam Dyer (University of Glasgow Photo Unit) for their valuable advice.

Open Access This article is licensed under a Creative Commons Attribution 4.0 International License, which permits use, sharing, adaptation, distribution and reproduction in any medium or format, as long as you give appropriate credit to the original author(s) and the source, provide a link to the Creative Commons licence, and indicate if changes were made. The images or other third party material in this article are included in the article's Creative Commons licence, unless indicated otherwise in a credit line to the material. If material is not included in the article's Creative Commons licence and your intended use is not permitted by statutory regulation or exceeds the permitted use, you will need to obtain permission directly from the copyright holder. To view a copy of this licence, visit http://creativecommons.org/licenses/by/4.0/. 


\section{References}

1. T.P. Campbell, Tapestry in the Baroque:threads of splendor. (Metropolitan Museum of Art New York, 2007)

2. A. Quye, et al., 'Wroughte in gold and silk': preserving the art of historic tapestries. (NMS Enterprises Limited-Publishing Edinburgh, 2009)

3. F. Lennard, M. Hayward, Butterworth-Heinemann series in conservation and museology (ButterworthHeinemann, Oxford, 2006), p. xxv

4. J.H. Hofenk de Graaff. (1997)

5. C.M. Breez, Textile Specialty Group Postprinrs. 75 (2000)

6. P. Duffus. University of Manchester (2013)

7. R. Allen, in Solutions The Influence of Locations on Treatments, edited by S. Howard. (London, 1999), p. 36

8. M.W. Ballard, in Lining and backing: the support of paintings, paper and textiles : papers delivered at the UKIC Conference, 7-8 November 1995. (United Kingdom Institute for Conservation of Historic and Artistic Works, London, UK, 1995)

9. E. Phipps, Looking at textiles: a guide to technical terms (J. Paul Getty Museum Los Angeles, Calif, 2011)

10. J. Alsayednoor et al., Strain 55, e12308 (2019)

11. D. Khennouf et al., Strain 46, 19 (2010)

12. F. Lennard, J.M. Dulieu-Barton, Stud Conserv. 59, 241 (2014)

13. L. Bratasz et al., Strain 51, 78 (2015)

14. D. Howell, in ICOM committee for conservation, 11th triennial meeting in Edinburgh, Scotland, 1-6 September 1996: Preprints. (Edinburgh, UK, 1996), p. 692

15. K. Frame et al., Stud. Conserv. 63, 87 (2018)

16. B.d. Chancel-Bardelot, in Consever et présenter les tapisseries: du bilan sanitaire à la mise en valeur. (Paris, 2015), p. 137

17. F. Lennard, Stud. Conserv. 51, 43 (2013)

18. A. Brutillot, in The Conservation of Tapestries and Embroideries, ed. by K. Grimstad (The Getty Conservation Institute, Brussels, Belgium, 1989), p. 75

19. C. Wild, A. Brutillot, in Tapestry Conservation Principles and Practice, ed. by F. Lennard, M. Hayward (Butterworth-Heinemann, Oxford, 2006), p. 177

20. K. Barker, Conservation news. 30 (2002)

21. R. Varoli-Piazza, Rosicarello R., and Giorgi M., in ICOM committee for conservation, 11th triennial meeting in Edinburgh, Scotland, 1-6 September 1996: Preprints. (1996), pp. 726

22. E.M. Catic. University of Glasgow (2019)

23. G. Trosbach. Technische Universitat Munchen (2002)

24. B. Pan, Meas. Sci. Technol. 29, 082001 (2018)

25. C. Gauvin et al., Strain 50, 428 (2014)

26. X. Zhao et al., Optik 171, 370 (2018)

27. S. Sharma, M. Sutcliffe, Compos. Part A Appl. Sci. Manuf. 35, 637 (2004)

28. P. Harrison, Compos. A Appl. Sci. Manuf. 81, 145 (2016)

29. A.A. Mercier, B.S. Jour, Research 5, 243 (1930)

30. R.R. Moorthy, P. Kandhavadivu, J. Text. Appar. Technol. Manag. 9, 1 (2015)

31. F. Hosseinali, J.A. Thomasson, Fibers 6, 49 (2018)

32. F. Lennard, R. Costantini, and P. Harrison, Stud Conserv. (2020)

33. S.J. Benson, Lennard F., and Smith M.J., in ICOM-CC 17th Triennial Conference Preprints. (Melbourne, Australia, 2014)

34. H. Sutherland and F. Lennard, Newsletter of the ICOM Committee for Conservation, Working Group of Textiles. 39 (2017)

35. M. Schön. Göteborgs Universitet (2017)

36. B. Pan et al., Meas. Sci. Technol. 20, 062001 (2009) 